\title{
О МЕТОДАХ ПОДКЛЮЧЕНИЯ МЕСТНЫХ СИНХРОННЫХ КОЛЕЦ К, ОСНОВНОМУ РАСПРЕДЕЛИТЕЛЮ СИСТЕМЫ ТЕЛЕМЕХАНИКИ *
}

\section{А. ГЕЛЬБ}

Основная задача телемеханики состоит в надежной передаче большого числа сигналов, команд по минимальному количеству линий связи. Увеличение емкости системы в различных устройствах решается по-разному, причем для этой цели разработаны весьма оригинальные и эффективные методы. Однако в некоторых случаях ряд новейших систем, построенных на бесконтактной аппаратуре, лнбо вообще не может пользоваться этими методами, либо их использование следует считать нецелесообразным.

На Всесоюзном совещании по телемеханике в мае 1958 г. в Ленинграде профессор М. А. Гаврилов высказал мысль об использовании для увеличения емкости систем телемеханики так называемых местных синхронных колец (фиг. 1), применимых как для бесконтактных, так и для контактных телемеханических устройств.

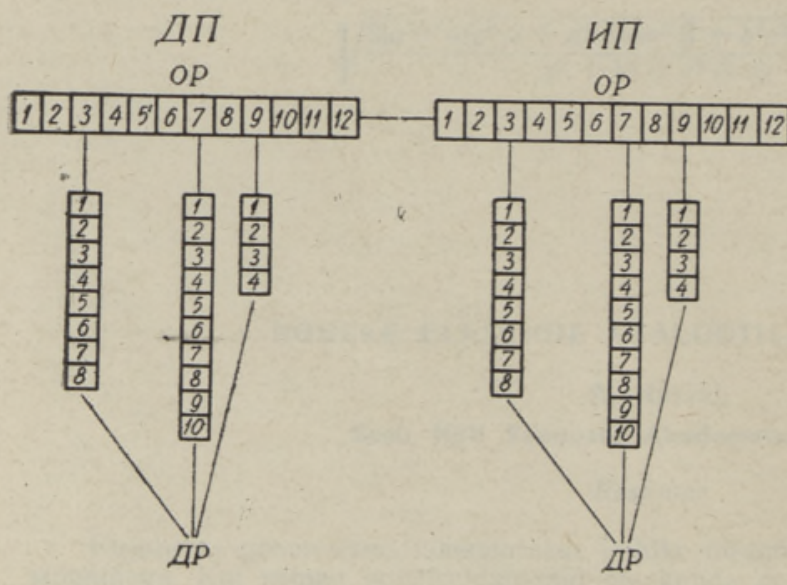

Фиг. 1.
При мечания

к фигурам.

1. На фиг. 1-7 использованы следующие обозначения:

дП - диспетчерский пункт

ИП - исполннтельный пункт

ОР - основной распределитель

ДР - дополнительный распредели тель

БС - блок связи

МК - местное кольцо

АПу - автоматическое программное устройств

БК - блок-контакты

ОУ - объекты управления

уу - устройство управления

УС - устройство снгнализацин

2. С целью упрощения схем на фиг. 1 и 6 опущены блоки связи н на всех фигурах - лннейные блокн.

При этом методе основные распределители системы телемеханики работают непрерывно. Все или часть управляемых объектов подключены к дополнительным распределителям-кольцам. В случае необходимости для управления или передачи информации с объектов либо по определенной, наперед заданной программе, либо вручную к основному распределителю подключаются местные кольца, в которых все

* Работа выполнена под руководством доктора технических наук М. А. Гаврилова (ИАТ АН СССР). 
объекты последовательно «опрашиваются» об их состоянии или передаются необходимые команды, а затем основной распределитель продолжает дальше «обегать» свои ячейки.

В настоящей статье приводятся различные методы подключения местных синхронных колец к основному распределителю и формулы для расчета максимального времени передачи сигналов при различных вариантах.

\section{СТРУКТУРА ПОДКЛЮЧЕНИЯ МЕСТНЫХ КОЛЕЦ К ОСНОВНОМУ РАСПРЕДЕЛИТЕЛЮ}

\section{I. Подключение всех объектов телеуправления, телесигнализации и телеизмерения только к местным кольцам}

Время одного цикла основного распределителя (фиг. 2) без местных колец выражается формулой

$$
T_{\text {ou }}=n_{\text {op }} t_{\mathrm{s}},
$$

где $T_{\text {оц }}$ - время одного цикла основного распределителя; $n_{\text {ор }}$ - количество ячеек в основном распределителе, обеспечивающих работу системы в целом и местных ко-

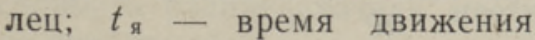
одной ячейки.

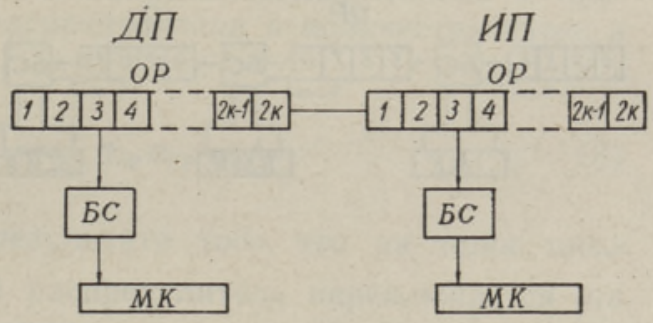

Фиг. 2.

1. Местное кольцо подключивается либо при уп равлении объектом или вызове опроса, либо при из енении объектом своего состояния и появлении с оответствующей сигнализаци (фиг. 3 ).

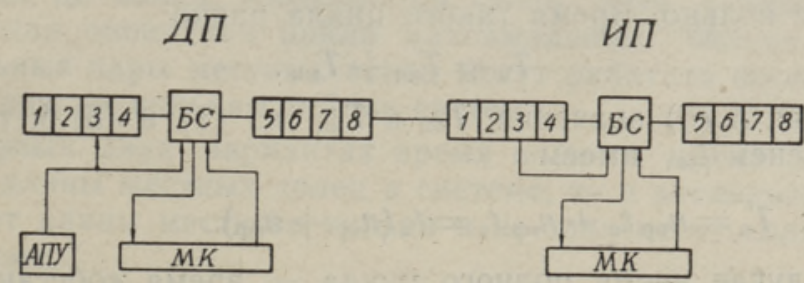

Фиг. 3.

В этом случае время цикла выражается формулой

$$
T_{\text {пц }}=T_{\text {оц }}+\kappa T_{\text {мц }}+\kappa t_{\text {св }} .
$$

Здесь $T_{\text {пц }}$ - время полного цикла распределителя, включая время «обегания» $\kappa$ местных колец; $T_{\text {мц }}$ - время местного кольца, где

$$
T_{\mathrm{M \mu}}=n_{\mathrm{Mp}} t_{\mathrm{g}} ;
$$

$n_{\text {м }}-$ количество ячеек местного кольца-распределителя; $t_{\text {св }}-$ время работы блока связи.

Поскольку в основном и местном распределителях используются однотипные ячейки, то время $t^{\text {я }}$ одинаково для обоих случаев. Тогда 


$$
\begin{gathered}
T_{\text {пц }}=T_{\text {оц }}+\kappa T_{\text {мц }}+\kappa t_{\text {св }}=n_{\mathrm{op}} t_{\mathrm{g}}+\kappa n_{\mathrm{Mp}} t_{\mathrm{g}}+\kappa t_{\mathrm{cB}}= \\
=t_{\mathrm{g}}\left(n_{\mathrm{op}}+\kappa n_{\mathrm{Mp}}\right)+\kappa t_{\mathrm{cB}} .
\end{gathered}
$$

Так как время $t_{\text {св }}$ либо входит во время работы основного распределителя, либо настолько мало (не больше времени работы одной ячейки), что им можно пренебречь, то считаем $t_{c в}=0$. В таком случае

$$
T_{\mathrm{nu}}=t_{\mathrm{g}}\left(n_{\mathrm{op}}+\kappa n_{\mathrm{mp}}\right) \text {. }
$$

В частном случае при $n_{\text {ор }}=n_{\text {мp }}=n_{\mathrm{p}}$

$$
T_{\mathrm{n \mu}}=t_{\mathrm{g}} n_{\mathrm{p}}(1+\kappa) \text {. }
$$

2. На каждом цикле «обегается»одно местное кольцо, на следующем цикле следующее и т. д. Последовательно «обегаются»все кольца (фиг. 4).
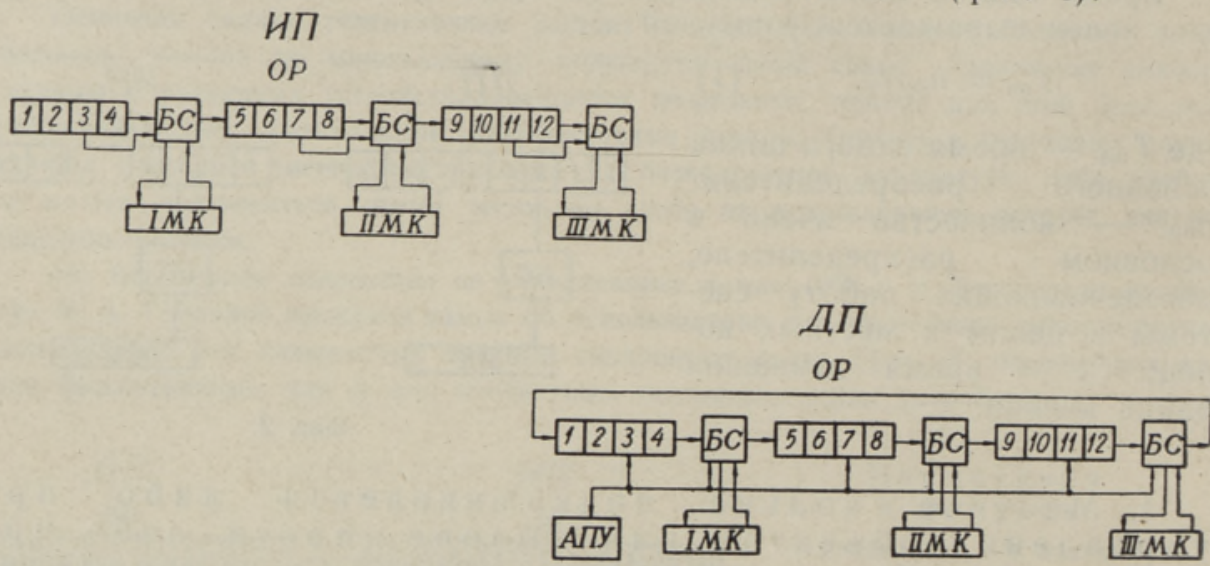

Здесь на одном цикле «обегается» весь основной распределитель и одно местное кольцо. Время такого цикла равно

$$
T_{\mathrm{u}}=T_{\text {оц }}+T_{\text {мц }} \text {. }
$$

Подставляя в (5) значения $T_{\text {оц и }} T_{\text {мц }}$ из (1) и (3) и также пренебрегая временем $t_{\text {св }}$, имеем

$$
T_{\mathrm{u}}=n_{\mathrm{op}} t_{\mathrm{s}}+n_{\mathrm{mp}} t_{\mathrm{g}}=t_{\mathrm{q}}\left(n_{\mathrm{op}}+n_{\mathrm{mp}}\right) .
$$

В этом случае время полного цикла - время «обегания» $\kappa$ местных колец в системе - выражается формулой

$$
T_{\text {пu }}=\kappa T_{\text {ц }}=\kappa t_{\mathrm{g}}\left(n_{\mathrm{op}}+n_{\mathrm{mp}}\right) .
$$

В обоих рассмотренных вариантах для подключения местных колец основной распределитель разрывался и в разрыв последовательно вклинивался местный распределитель-кольцо.

Далее рассмотрим вариант, при котором нет необходимости изменять структуру основной схемы: местное кольцо подключается параллельно основному.

3. На каждом цикле основного распределителя к нему подключают со всех местных колец одну ячейку для передачи команд телеуправления и одну - для передачи с нее телесигнализации (фиг. 5). 


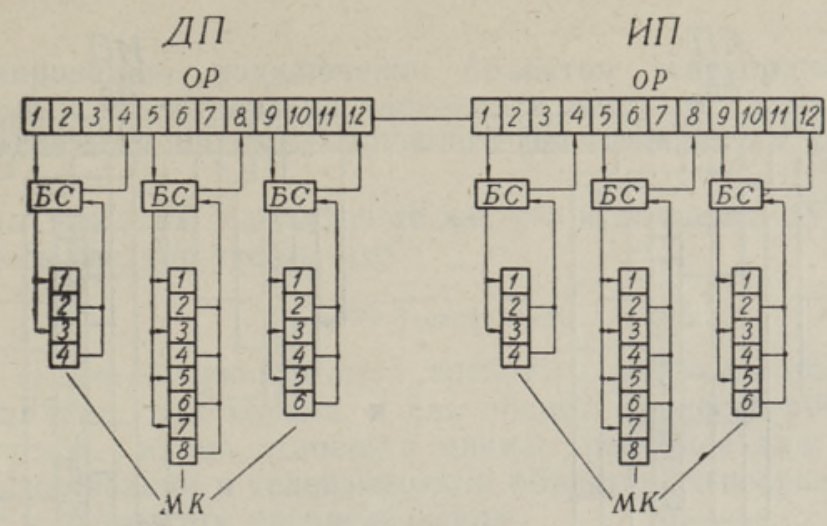

Фиг. 5.

Здесь время полного «обегания» всех местных колец зависит от продолжительности цикла основного распределителя и количества ячеек в наибольшем местном кольце, т. е.

$$
T_{\text {пц }}=T_{\text {оц }} \frac{n_{\text {мp } \max }}{2}=\frac{1}{2} n_{\text {op }} n_{\text {мp мax }} t_{\mathrm{s}} .
$$

Коэффициент $\frac{1}{2}$ появляется в результате того, что за один цикл основного распределителя местный распределитель переключается на две ячейки.

В общем случае:

$$
T_{\mathrm{nu}}=\frac{1}{2} n_{\mathrm{op}} n_{\mathrm{mp}} t_{\mathrm{q}} .
$$

Местные кольца, имеющие меньшее количество ячеек, чем максимальное, дойдя до конца, запускаются снова. Они начинают новый цикл, не ожидая окончания цикла максимального кольца. Следовательно, отдельные пары местных колец могут работать несинхронно по отношению к другим кольцам той же системы.

Если в первых двух вариантах время полного цикла зависело от количества и длины местных колец в системе, то в последнем варианте оно зависит от длины местного кольца и не зависит от количества таковых в системе.

4. При параллельном подключении местного кольца на каждом цикле распределителя «обегается»не одна, а две, три и т. д. п а я чеек (фиг. 6).

При этом варианте на основном распределителе дополнительно используются одна, две и т. д. пар ячеек на каждое местное кольцо (число их зависит от кратности дополнительных пар ячеек, работающих на одном цикле).

На схеме (фиг. 6) показано два местных кольца, из которых первое за один цикл основного распределителя выдает сигналы и принимает команды от двух, а второе - от трех пар ячеек. Первое кольцо, состоящее из восьми ячеек, выдает и принимает информацию за два цикла основного распределителя, а второе кольцо, состоящее из 18 ячеек, за три цикла основного распределителя. 


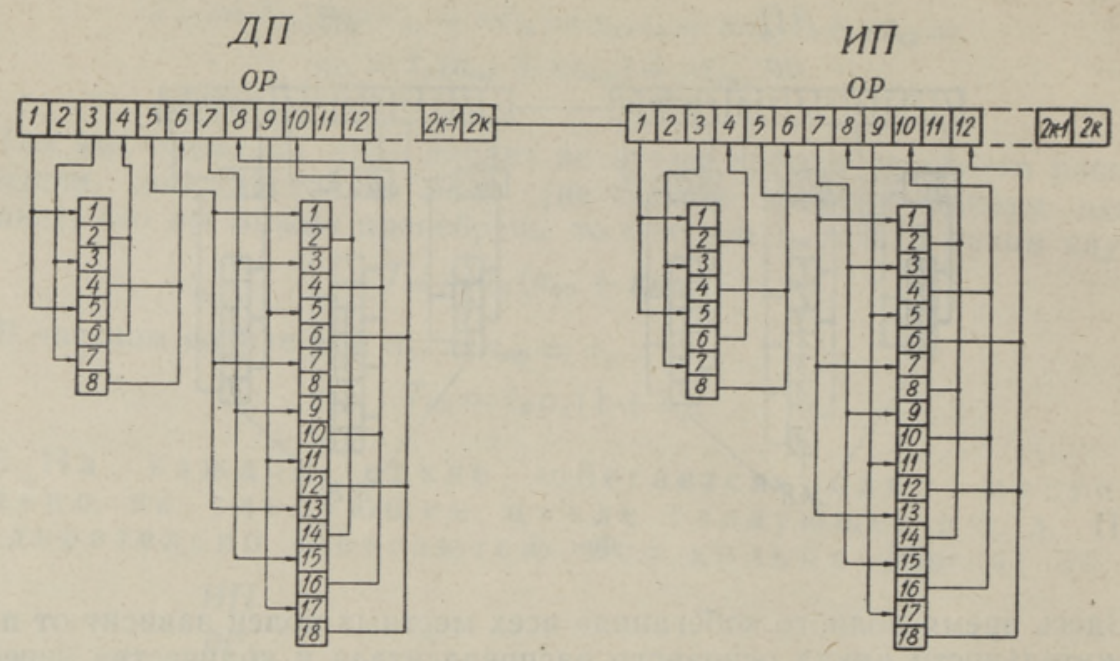

Фиг. 6.

Формула полного цикла в этом случае имеет вид:

$$
T_{\text {mu }}=\frac{1}{2+a} n_{\text {op }} n_{\text {mp }} t_{\mathrm{s}}+T_{\text {oul } 1},
$$

где $\alpha$ - количество дополнительных ячеек, с которых информация выдается и принимается на том же цикле, что и с первой пары ячеек, а под

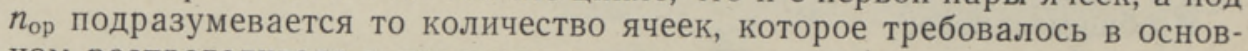
ном распределителе в предыдущем варианте. вид

В этом случае значение $T_{\text {оц }}$ в отличие от формулы (1) приобретает

$$
T_{\text {ou1 }}=2 \alpha \kappa t_{\mathrm{s}}
$$

и выражает время, затрачиваемое на «обегание» дополнительных ячеек основного распределителя, включенных для передачи и приема информации с дополнительных пар ячеек (фактически последние входят в состав основного распределителя, а время $T_{\text {оц1 }}$ является составной частью $\left.T_{\text {оц }}\right)$.

Формулы (10) и (11) справедливы для случая, когда со всех $\kappa$ местных колец информация выдается и принимается с одинакового количества ячеек.

Если же количество ячеек, работающих на одном цикле основного распределителя, разное, то формула (10) имеет вид:

$$
T_{\mathrm{nu}}=\frac{1}{2+a_{\min }} n_{\mathrm{op}} n_{\mathrm{mp}} t_{\mathrm{s}}+T_{\text {ou1 }},
$$

где $\alpha_{\min }-$ минимальное количество дополнительных ячеек, подключаемых к передаче на одном цикле, т. е. $\alpha$ для кольца, требующего максимального числа циклов;

$$
T_{\text {ou1 }}=2 \kappa t_{\mathrm{g}}\left(\alpha_{1}+\alpha_{2}+\alpha_{3}+\ldots+\alpha_{k}\right) ;
$$

$\alpha_{1}, \alpha_{2}, \alpha_{3}, \alpha_{k}-$ коэффициенты $\alpha$ для $1,2,3, \ldots \kappa$-го местных колец. 
II. Комбинированное подключение объектов телеуправления, телесигнализации и телеизмерения частично к местным кольцам, частично к выделенным ячейкам основного распределителя (фиг. 7)

Формулы для этих вариантов те же, что и для предыдущих случаев Однако, здесь следует учесть, что

$$
T_{\text {ои }}=T_{\text {оц } 2}+T_{\text {оц3 }},
$$

где $T_{\text {оц2 }}$ - время основного цикла, требуемое для «обегания» ячеек, обслуживающих местные кольца, и для общего процесса работы распределителей; $T_{\text {оц }}-$ время основного цикла, требуемое для телеуправления, телесигнализации и телеизмерения объектов, непосредственно подключенных к основному распределителю.

\section{ДП}

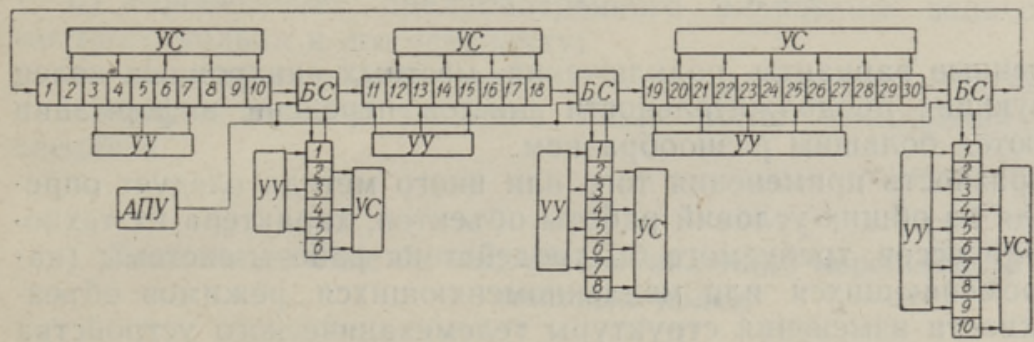

ИП

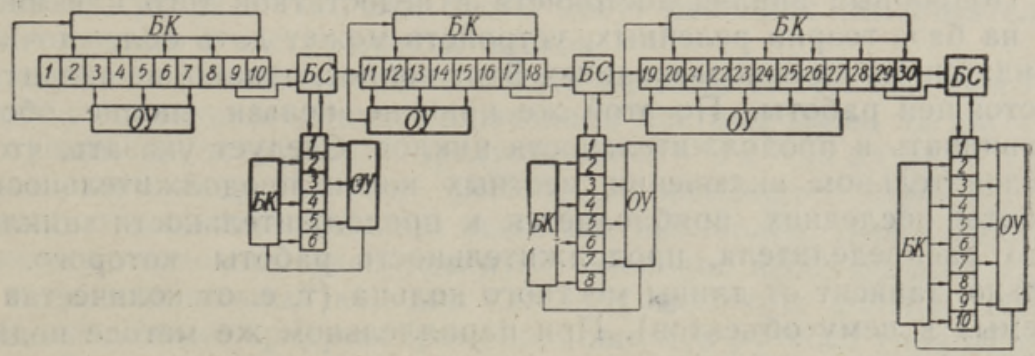

Фиг. 7.

Формулы продолжительности циклов приведенных вариантов подключения местных колец в случае подключения объектов также к основному распределителю приобретают следующий вид:

1. Дополнением формулы (4) получаем:

$$
T_{\mathrm{mu}}=T_{\text {оц }}+\kappa T_{\text {мц }}=T_{\text {оц } 2}+T_{\text {оц } 3}+\kappa T_{\text {мц }}=n_{\text {ор }} t_{\mathrm{g}}+n_{\text {ук }} t_{\mathrm{g}}+\kappa n_{\mathrm{Mp}} t_{\mathrm{g}},
$$

откуда

$$
T_{\mathrm{nu}}=t_{\mathrm{s}}\left(n_{\mathrm{om}}+n_{\mathrm{yk}}+\kappa n_{\mathrm{Mp}}\right) .
$$

Здесь $n_{\text {ук }}$ - количество дополнительных ячеек основного распределителя, предназначенных для непосредственного подключения объектов управления и контроля; $n_{\text {ом }}$ - количество ячеек основного распределителя, обеспечивающих общий процесс его нормальной работы, а также подключение и работу местных колец. В вариантах первой серии $\boldsymbol{n}_{\text {ом }}=\boldsymbol{n}_{\text {ор- }}$ 
2. Исходя из формулы (7), для данного варианта

$$
T_{\mathrm{nu}}=\kappa t_{\mathrm{s}}\left(n_{\mathrm{om}}+n_{\mathrm{y \kappa}}+n_{\mathrm{mp}}\right) .
$$

3. Из формулы (9) получаем:

$$
T_{\mathrm{nц}}=\frac{1}{2}\left(n_{\mathrm{om}}+n_{\mathrm{yK}}\right) n_{\mathrm{Mp}} t_{\mathrm{s}} .
$$

4. На основании формулы (12) получаем:

$T_{\mathrm{n \mu}}=\frac{1}{2+\alpha_{\min }}\left(n_{\mathrm{om}}+n_{\mathrm{y \kappa}}\right) n_{\mathrm{mp}} t_{\mathrm{g}}+2 \kappa t_{\mathrm{g}}\left(\alpha_{1}+\alpha_{2}+\alpha_{3}+\ldots+\alpha_{\mathrm{k}}\right)$.

В случае $\alpha_{1}=\alpha_{2}=\alpha_{3}=\ldots=\alpha_{\mathrm{k}}$ имеем:

$$
T_{\mathrm{nц}}=\frac{1}{2+a}\left(n_{\mathrm{om}}+n_{\mathrm{y \kappa}}\right) n_{\mathrm{mp}} t_{\mathrm{s}}+2 \kappa a t_{\mathrm{s}} .
$$

Рассмотренные варианты подключения местных синхронных “колец и соответствующие продолжительности циклов передачи информации характеризуются большим разнообразием.

Целесообразность применения того или иного метода следует определять, исходя из общих условий работы объектов, характера их технологических процессов, требуемого быстродействия работы системы (наличия быстроменяющихся или медленноменяющихся режимов объектов), возможности изменения структуры телемеханического устройства (в случае подключения местных колец к уже работающим устройствам) и т. д.

Подробный анализ достоинств и недостатков того или иного метода на базе теории релейных устройств может дать более точные рекомендации шо их использованию. Этот вопрос, однако, не входит в задачи настоящей работы. По этой же причине нельзя вполне обоснованно сравнивать и продолжительности циклов. Следует указать, что при последовательном включении местных колец продолжительность. цикла работы последних прибавляется к продолжительности цикла основного распределителя, продолжительность работы которого, следовательно, зависит от длины местного кольца (т. е. от көличества пөдключаемых к нему объектов). При параллельном же метөде подключення местных колец продолжительность основного цикла зависит только от длины основного распределителя и не зависит от длины местных колец.

Надежность работы синхронных колец зависит, во-первых, от используемой аппаратуры и, во-вторых, от структуры построения системы в целом. В общем случае надежность работы местных колец сводится к надежности работы всей системы.

\section{Требования, предяъвляемые к блокам связи}

В общем случае блок связи. должен обеспечить:

1) подключение местного кольца к основному распределителю в предусмотренный схемой момент или по указанию диспетчера;

2) передачу команд управления на объекты, подключенные к кольцу, и информацию от них на диспетчерский пункт;

3) бесперебойную работу основного распределителя при отключенном местном кольце. 
Кроме того, отдельные варианты подключения предъявляют ряд дополнительных требований.

При последовательном включении местного кольца в основной распределитель блок связи, кроме вышеприведенных функций, должен еще обеспечить:

а) разрыв цепи основного распределителя;

б) запуск местного кольца от последнего элемента первой части основного распределителя;

в) запуск второй части основного распределителя от последнего элемента местного кольца;

г) прямое соединение первой части основного распределителя с его второй частью в случае исключения местного кольца.

При параллельном подключении местных колец блок связи, кроме общих требований, должен обеспечить:

а) запуск местного кольца от какого-то элемента основного распределителя;

б) «пробегание» предусмотренного вариантом количества шагов местного кольца и его остановку;

в) последовательную работу всех элементов местного кольца, т. е. запуск первого элемента лишь после завершения работы последнего.

\section{Дополнительные блоки, обеспечивающие нормальную работу местных колец}

В связи с подключением местных колец к основным распределителям систем телемеханики возникает необходимость в некоторых дополнительных блоках, узлах и т. д. Эти блоки могут быть как самостоятельными, так и входить в состав других блоков.

Первым обязательным блоком является названный блок связи.

В схемах с последовательным включением местных колец на диспетчерском пункте должно быть предусмотрено а в том а ти еское программное устройство (АПУ), управляющее порядком и периодичностью включения тех или иных местных колец. Для передачи этой команды на основном распределителе очевидно необходимо предусмотреть один специальный код для каждого местного кольца. АПУ (фиг. 2) должно также предусмотреть возможность ручного управления в случае необходимости.

В некоторых случаях (вариант 1) управление включением местных колец со стороны диспетчерского пункта может быть совмещено с управлением объектами. В таком случае на исполнительном пункте должен быть предусмотрен блок за п асания информ ц и и, который в случае возникновения какой-либо информации на исполнительном пункте «запрашивает» с диспетчерского пункта команду о подключении местного кольца. Такая команда может быть выдана автоматически или вручную. Кроме того, блок запасания должен сохранить возникшую информацию и передать ее на диспетчерский пункт тогда, когда для этого представится возможность, т. е. когда местное кольцо будет подключено.

Для телеизмерения необходимо предусмотреть блок вызов а т ел е и ме рения. Этот вызов тоже может быть как автоматическим, так и ручным.

В схемах параллельного подключения местных колец все необходимые функции выполняются блоком связи.

3 TA Toimetised T-2 61 


\title{
Выводы
}

1. Рассмотренные варианты подключения местных синхронных колец к основному распределителю системы телемеханики свидетельствуют об их многообразии и гибкости.

2. Для более точного сравнения различных методов необходимо провести структурный анализ схем на базе теории релейных устройств.

3. При последовательном включении местных колец продолжительность основного цикла зависит от их длины, а при параллельном подключении - не зависит от нее.

4. При прочих равных условиях предпочтение следует отдавать параллельному методу подключения синхронных колец, ибо в этом случае, во-первых, нет необходимости изменять структуру устройства, во-вторых, не удлиняется цикл работы основного распределителя и, в-третьих, не требуется отдельного кода для подключения местного кольца. Кроме того при параллельном подключении требуется меньше дополнительных блоков, а необходимый блок связи имеет более простую структуру, чем соответствующий блок при последовательном соединении.

5. В случае наличия весьма важных объектов с быстроменяющимися характеристиками следует пользоваться комбинированным методом подключения как к местным кольцам, так и к выделенным ячейкам основного распределителя. К последним и следует подключать объекты с быстроменяющимися характеристиками.

6. В случае необходимости можно в одной и -той же системе пользоваться различными вариантами подключения местных колец.

Принципиальные электрические схемы вышеуказанных блоков и их характеристики будут опубликованы в специальной статье.

Институт кибернетики Академии наук Эстонской ССР

Поступила в редакцню 26. XI 1960

\section{KOHALIKE SUNKROONRONGASTE LULITAMISE MEETODEIST TELEMEHAANIKA SUSTEEMI JAOTAJASSE}

\author{
A. Gelb \\ Resümee
}

Artiklis vaadeldakse telemehaanika süsteemi poolt teenindavate objektide arvu suurendamise võimalust nn. kohalike rōngaste abil. Viimased lülitatakse süsteemi ja neilt saadetakse osa informatsiooni teatud perioodilisusega edasi sidekanalisse. Artiklis esitatakse rida vōimalikke variante ning tuletatakse vastavate variantide tsükli kestusvalemid. Ka on välja selgitatud rea uute blokkide, nagu sidebloki, automaatprogrammseadme jne. loomise vajadus ning käsitletakse iga bloki tööd.

Käsitletud variantidest tuleks eelistada kohalike rõngaste paralleelset sidestust süsteemiga; see ei nôua süsteemi struktuuri muutmist ega avalda mōju süsteemi töötsükli kestusele. On esitatud ka variantide struktuuri skeemid.

Eesti NSV Teaduste Akadeemia

Küberneetika Instituut
Saabus toimetusse

26. XI 1960 


\section{UBER DIE METHODEN DER EINSCHALTUNG ZUSÄTZLICHER SYNCHRONISIERT UMSCHALTENDER RINGE IN FERNLENKUNGSSYSTEMEN}

A. Gelb

\section{Zusammenfassung}

Der Artikel behandelt die Möglichkeit einer Vergrösserung der Zahl der von einem Fernlenkungssystem bedienten Objekte durch Einschaltung zusätzlicher Ringe, die einen Teil der Information mit einer gewissen Periodizität in den Verbindungskanal weiterleiten. Der Artikel bringt eine Reihe Varianten, auch werden Formeln für die Bestimmung der Dauer der entsprechenden Zyklen abgeleitet. Zugleich wird die Notwendigkeit der Anwendung verschiedener neuer Einrichtungen hervorgehoben, wie z. B. des Verbindungsblocks, der automatischen Programmeinrichtung. usw., und es wird die Arbeit dieser Einrichtungen erläutert.

Von den betrachteten Varianten sollte die Paralleleinschaltung der Synchronringe in das System vorgezogen werden; die Struktur des Systems braucht dabei nicht geändert zu werden, auch bleibt die Dauer des Arbeitszyklus dieselbe. Ferner bringt der Artikel die Strukturschemas der einzelnen Varianten.

\section{Institut für Kybernetik}

der Akademie der Wissenschaften der Estnischen SSR
Eingegangen am 26. Nov. 1960 